\title{
MICROSTRUCTURE AND THE PULSAR RADIO EMISSION MECHANISM
}

\author{
VALENTÍN BORIAKOFF
}

Worcester Polytechnic Institute

\begin{abstract}
The properties of pulsar radio pulse microstructure are reviewed, then consideration is given on how, in the frame of the Ruderman-Sutherland pulsar model, an emission mechanism can be devised which explains many of the known characteristics of micropulses and of subpulses.
\end{abstract}

\section{Introduction}

Pulsar radio pulse microstructure is described as an emission phenomenon of a short characteristic time scale, typically up to the order of a few hundreds of microseconds. It is also frequently extremely intense emission, of over $10^{3} \mathrm{Jy}$, making it the most intense component of a radio pulse. A high but variable degree of polarization is apparent. Micropulses occasionally come in nearly periodic sequences (quasi-periodic, but not to be confused with X-ray pulsar quasi-periodicities). Micropulses are therefore important in the study and interpretation of the radio emission mechanism, specifically, how can the mechanism produce the three observed types of pulsar radioemission: microstructure, subpulses and amorphous emission (Cordes 1975).

\section{Microstructure properties}

Microstructure in radio pulses was first seen by Craft, Comella and Drake (1970). Hankins (1971) first published an observation of a pulse where a quasi-periodic sequence of micropulses is obvious. Boriakoff (1973) showed that the averaged correlation function (ACF) showed a statistically significant feature representing a periodicity of $0.9 \mathrm{~ms}$ period in PSR 2016+28. No ACF feature at $1.8 \mathrm{~ms}$ was found, indicating that either the microstructure periodicity is a low $Q$ process, or that the micropulses appear predominantly in pairs or singly, and that triplets and multiplets were rare. It was also noted that occasionally a regular periodic microstructure sequence might be interrupted and restarted anew with the same period but at different phase. Quasi-periods exist in the $0.2-2 \mathrm{~ms}$ range, independent of the pulsar period. Occasionally more than one quasi-periodic sequence coexists simultaneously (e.g. in data of Ferguson and Seiradakis 1978).

Microstructure has a specific time length, characteristic time, which statistically indicates a "maximum" time length of the envelope of micropulses, although individual micropulses often have smaller time widths. The characteristic time scale, $\tau_{\mathrm{c}}$, was defined by Hankins (1972) as the ACF lag where the slope changes sharply. Some individual micropulses are longer than $\tau_{c}$, and the distribution of time widths shows small contributions of micropulses of medium and small time lengths compared to $\tau_{c}$ (Popov, Smirnova, and Soglasnov 1987).

$\tau_{c}$ ranges from a few hundreds of microseconds to $\sim 1 \mathrm{~ms}$, and is independent of the observing frequency (Boriakoff 1983). The ACF characteristic time scale feature is used as an indicator of microstructure.

Occasionally two values of $\tau_{\mathrm{c}}$ coexist. For example, Soglasnov, Popov and Kuz'min (1983) found $\tau_{\text {rmcl }}=600 \mu \mathrm{s}$ and $\tau_{\mathrm{c} 2}=50 \mu \mathrm{s}$ in low frequency data of PSR 1133; $\tau_{c 1}$ is known to exist at higher frequencies.

Not all pulsars have microstructure. Some, like PSR 0301+19, seem not to have it at all. Other pulsars like PSR 2016+28 have different spectral indices of microstructure in the two average profile peaks (Boriakoff 1983). Because microstructure auto- and cross-correlation function shapes are similar it is inferred that the spectrum and intensity distribution of all micropulses at a certain pulse longitude is the same (Boriakoff 1983). For PSR 0950+08 microstructure appears in the main pulse with $\tau_{c}=130 \mu \mathrm{s}$ and in the interpulse with $\tau_{\mathrm{c}}=79 \mu \mathrm{s}$.

Microstructure emission is frequently associated with subpulse emission, that is, on many occasions on those longitudes where there is subpulse emission, superimposed micropulses can be found. Microstructure is wideband (Rickett, Hankins, and Cordes 1975, Boriakoff 1976), and at different observing frequencies each micropulse is located at the same pulse longitude with respect to the pulse fiducial point (Boriakoff 1976). Hence, after propagation through the interstellar medium the microstructure pulses follow the cold plasma dispersion law regardless of their location in longitude. However, one observation seems to show that at low frequencies PSR $1133+16$ microstructure fol- 
lows two slightly different cold plasma dispersion laws according to where the microstructure is located in pulse longitude (Popov, Smirnova, and Soglasnov 1987). In contrast to subpulses and other pulsar emission whose location in longitude changes with the observing frequency, indicating an instantaneously narrowband process, microstructure is truly wideband, that is, a micropulse appears simultaneously over a wide range of frequencies. Microstructure bandwidths of $i 1 \mathrm{GHz}$ (Boriakoff and Ferguson 1981) are observed, but some pulsars like PSR $1133+16$ show decorrelation bandwidths smaller than the frequency range over which the microstructure is observed. In adjacent bandwidths microstructure cross-correlates highly, but it does not correlate between above and below $1.2 \mathrm{GHz}$ (Boriakoff and Ferguson 1981, Popov, Smirnova, and Soglasnov 1987). This frequency corresponds to a change in the properties of the average pulse profile (Seiber, Reinecke, and Wielebinski 1975).

The

microstructure narrowband spectrum shows deep modulation (Boriakoff 1973, Cordes and Hankins 1979), which accounts for some of the variability of the micropulse structure when observed at two different frequencies simultaneously. Ferguson and Seiradakis (1978) have shown that the polarization behavior of PSR $1133+16$ micropulses at frequencies above $1.7 \mathrm{GHz}$ is of two types: the linear polarization angle may stay constant within the time of one micropulse, changing only at the micropulse edges, or it may be changing with time more or less linearly inside a micropulse; angle swings of up to $60^{\circ}$ are noted. They also show that the polarization angle during an intense micropulse may be independent of that of the surrounding amorphous or subpulse emission. Cordes and Hankins (1977) have shown that at $430 \mathrm{MHz}$ for the same pulsar the polarization angle predominantly remains constant within the micropulse, changing only at the edges. The type of pulses chosen for the study (quasi-periodic micropulse sequences with no or little additional radiation in the first case, mixed emission with single micropulses in the second) and the different observed radio frequency may account for the difference in results.

Microstructure has a steep spectrum, steeper than that of subpulses and of that of the main pulse itself. In PSR $1133+16$ the microstructure spectral indices between 318 and $196.5 \mathrm{MHz}$ are -2.1 in the first peak and -10 in the second peak.

\section{Interpretation and model}

To accommodate the variety of microstructure phenomena described above and the characteristics of the subpulse and amorphous emission, Boriakoff and Ferguson (1981), and Boriakoff (1983) proposed an emission mechanism composed of two parts: a narrowband emitting mechanism that would produce subpulses and other emission which follows the increasing pulse width and emission separation from the fiducial point with lower observing frequency, and a wideband mechanism that would instantanously produce wideband micropulses. The difference between the two mechanisms is the coherence length of the emitting bunches. In Boriakoff (1983) from the possible plasma configurations in the emitting zone a choice is made for the thin filament or flux tube (less than $1 \mathrm{~km}$ diameter for PSR 1133+16), one per each micropulse, the outside dimensions of which will determine the maximum time width of the micropulse. Inside this flux tube single charge bunches of coherence length less or equal to $\lambda / 2$ of the critical frequency corresponding to that emission radius above the star will emit a wideband flash of radiation as it sweeps by the observer. The time limitation of the micropulses is fulfilled, i.e. the flux tube maps to the observed time width by stellar rotation, and the radiation, composed of a multitude of individually coherent bunches adding incoherently, has a wide instantaneous bandwidth.

A wider flux tube is postulated for the subpulses in which particle bunching also exists, but the emitting structure is not a single bunch but a set of bunches radiating coherently together. The coherence length over the bunch set is several wavelengths, which prompts a limitation on the bandwidth of the emitted radiation: only in a small range of wavelengths is coherence effective over the whole set of bunches. This is then a band-limited radiator working effectively only in the frequencies surrounding a critical frequency. If this critical frequency depends upon the height above the neutron star (radius-to-frequency mapping) this process will provide an interpretation of the observable properties of subpulses: a radiation process intrinsically narrowband but actually extending over a wide range of frequencies through the physical agency of emitting structures inside a wide emitting magnetic flux tube, all emitting at the critical frequency corresponding to their particular radius. Hence, just by changing the coherence length, the emission of bunched particles is sufficient to approximate both the microstructure and the subpulse emission. Polarization properties are fulfilled to a large extent, too, for radiation emitted in the narrow (microstructure) flux tube has a linear polarization position angle in the plane containing the magnetic field line. Because the microstructure flux tube is thin, this plane has nearly the same angle for all points inside the flux tube, hence, the emission 
within a micropulse would have a nearly constant linear polarization angle. This fulfills the condition that the microstructure has largely a constant polarization angle within the micropulse.

In the case of the wide flux tube (subpulse and amorphous emission) the polarization angle follows the general change of angle through the pulse (Cordes and Hankins 1977), again fulfilling the subpulse requirements. The cross-section of the wide flux tube is probably kidney-shaped since it represents similar conditions (including a similar magnetic field line radius of curvature) in the crosssection of the polar cap magnetic field.

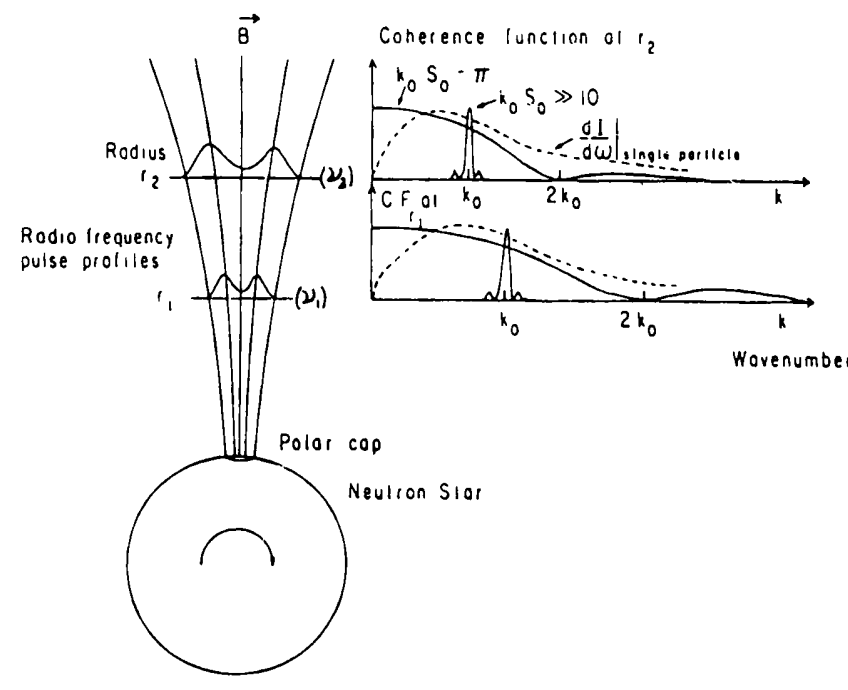

Figure 1 Two graphs are shown at two radii over the surface of the neutron star. At each radius the average pulse profile of a clouble-peaked pulse pulsar is also drawn. In each graph the dotted-line spectrum shows a single particle emission spectrum function of wavenumber $(k=1 / \lambda)$. On both graphs there are two coherence functions: one for a single bunch $\left(k_{0} S_{0}=\pi\right)$ and another for a set of bunches radiating coherently $\left(k_{0} S_{0} \gg 10\right)$ where $k_{0}$ is the critical frequency, and $S_{0}$ is the physical length of the coherentlyradiating plasma. The product of a coherence function and the single particle radiation spectrum produces the actual radiated spectrum.

The micropulses will be generated with the bulk of the spectral emission of the single bunches at frequencies under $2 k_{0}$. At a given observing frequency a micropulse will have a spectrum starting at a frequency higher than the observing frequency and ending somewhere at low frequencies. Throughout the spectrum the time width and location with respect to the fiducial point, will remain the same. In dual frequency observations some micropulses will have $2 k_{0}$ at a frequency in between the observing frequencies, hence there should be a preponderance of microstructure at lower frequencies, as observed. However, a slight increase in characteristic width should be present for microstructure generated with lower $2 k_{0}$ (larger radius), due to the changing geometry of the flux tube. This may be counteracted by the same mechanism that maintains the width of the subpulses practically independent of frequency.

In the Ruderman-Sutherland (1975) pulsar model highly energetic ions extracted from the neutron star surface, and positrons created and accelerated in the polar cap gap are injected in the plasma column existing above the polar cap. This plasma is created by a cascade of electron-positron pairs and $\gamma$ radiation started by the energetic positrons. The movement of the highly energetic ions and positrons $\left(\gamma \leq 10^{6}\right)$ through the relatively slow electronpositron plasma $\left(\gamma_{ \pm} \approx 800\right)$ produces a two-stream instability, which tends to produce particle bunching. As the electron-positron plasma moves along the curved magnetic field lines the particles radiate by the synchrotron process, with a critical frequency at the peak of the spectrum given by

$$
\omega_{c}=\frac{3}{2} \gamma_{ \pm}^{3} \frac{c}{\rho}
$$

where $\rho$ is the radius of curvature of the magnetic field line. The plasma frequency $\omega_{p}$ determines the frequency at which the two stream instability bunching grows

$$
\omega_{\mathrm{p}}=2\left(\frac{2 \gamma_{\max } e \Omega B R^{3}}{m c r^{3}}\right)^{1 / 2}
$$

where $\gamma_{\max }$ is the maximum attained by the particles at the top of the polar cap gap, $R$ is the neutron star radius, $r$ is the radiation radius, and $\Omega$ is the pulsar rotation frequency. When $\omega_{\mathbf{c}} \approx \omega_{\mathrm{p}}$ then the bunching is effective and radiates very effectively. This happens best in the outside area of the polar magnetic field (greater curvature of the magnetic field lines), corresponding to the two-peak "cone" emission (Rankin 1983a). For a typical pul$\operatorname{sar} \Omega=6 s^{-1}, B=10^{12} G, r=10^{2} R=10^{8} \mathrm{~cm}$, and $\gamma_{\max }=10^{6}$ then $\omega_{\mathrm{p}} \approx 10^{10} \mathrm{~s}^{-1}$, approximately $1 \mathrm{GHz}$. In the radiation zone $\gamma \approx 10^{\circ} \mathrm{cm}$, it follows that the synchrotron radiation frequency is $\omega_{\mathrm{c}} \approx 10^{11} \mathrm{~s}^{-1}$, hence $\omega_{\mathrm{p}} \approx \omega_{\mathrm{c}}$, favorable conditions for radiation.

A relativistic particle going along a curved path of curvature $\rho$ produces a radiation flash to a stationary observer of time length (in the observer's frame)

$$
\Delta t \approx \frac{1}{\gamma^{3}} \frac{\rho}{c}
$$

for $\gamma \approx 10^{3}$ and $\rho \approx 10^{\circ} \mathrm{cm}$ results in a $\Delta t \approx$ $10^{-10} \mathrm{~s}$, of the order of a nanosecond. Hence the spectrum of such a flash will extend to $1 / \Delta t \approx$ $1 \mathrm{GHz}$. Buschauer and Benford $(1976,1980)$ give the expression for the radiation of a bunching plasma column of wavenumber $k_{0}$, coherence length $S_{0} \ll \rho$, radial thickness $\xi_{0} \ll \rho / \gamma^{2}$, and of vertical dimension $\eta_{0} \ll \rho$ moving along a curved path of 
curvature $\rho$ as (per unit solid angle, per unit frequency)

$$
\left.\frac{d^{2} I}{d \Omega d \omega}\right|_{c}=\left.\frac{\left(\sigma_{0} S_{0} \xi_{0} \eta_{0}\right)^{2}}{4 q^{2}}(C F) \frac{\sin ^{2}\left(k \eta_{0} \theta / 2\right)}{\left(k \eta_{0} \theta / 2\right)^{2}} \frac{d^{2} I}{d \Omega d \omega}\right|_{\mathrm{sp}}
$$

where $\Omega$ is the solid angle, $\theta$ is the angle, $C F$ is the coherence factor and $d^{2} I /\left.d \Omega d \omega\right|_{\mathrm{sp}}$ is the single particle radiation intensity expression (Jackson 1962). $\sigma_{0}$ is the amplitude of the charge density perturbation. The term $\left[\sin ^{2}\left(k \eta_{0} \theta / 2\right)\right] /\left(k \eta_{0} \theta / 2\right)^{2}$ is a diffraction term due to the finite dimensions of the radiating face of the plasma column. The longitudinal coherence factor is

$$
C F=A^{2}+B^{2}-2 A B \cos \left(k_{0} S_{0}\right)
$$

where

$$
\begin{aligned}
& A=\frac{\sin \left[\left(k-k_{0}\right) S_{0} / 2\right]}{\left[\left(k-k_{0}\right) S_{0} / 2\right]} \\
& B=\frac{\sin \left[\left(k+k_{0}\right) S_{0} / 2\right]}{\left[\left(k+k_{0}\right) S_{0} / 2\right]}
\end{aligned}
$$

The coherence factor $C F$ is plotted in figure 1 for two different coherence lengths $S_{0}$ resulting in narrowband and broadband emission.

Buschauer and Benford consider the emission in a pulsar governed by one single value of coherence length. However, the assignment of long coherence lengths to subpulses and short coherence lengths to microstructure in the magnetosphere of a single pulsar is of more interest.

This physical description does not conflict with the Amplitude Modulated Noise model (Rickett 1975). It provides a framework for the determination of Rickett's $a(t)$ function. Gil (1981) presented a similar model of microstructure emission partly based on the work of Boriakoff (1983). However, he postulated microstructure emission inside a thin flux tube that is narrowband and that follows radius to frequency mapping, hence the longitude location of the same micropulse observed at different frequencies has to follow the same separation from the fiducial point with observing frequency as the subpulses and other emission do, for example for PSR 1133+16 a micropulse located $15 \mathrm{~ms}$ from the fiducial point at $318 \mathrm{MHz}$ would be located $17 \mathrm{~ms}$ from the fiducial point at $196.5 \mathrm{MHz}$, however, this is not observed (Boriakoff 1983).

\section{Conclusions}

We have used the approach of Buschauer and Benford $(1976,1980)$ for the description of the radiation of a plasma column radiating coherently extending it to interpret physical entities present in the pulsar radio radiation: microstructure and subpulses. Observational facts are compared to conclusions derived from the model and in general agree well. 